\title{
An exploratory study of the association between SORLI polymorphisms and sporadic Alzheimer's disease in the Han Chinese population
}

This article was published in the following Dove Press journal:

Neuropsychiatric Disease and Treatment

12 June 2015

Number of times this article has been viewed

\section{Feng Zhang ${ }^{1,2, *}$ \\ Xiaowei $\mathrm{Liu}^{2, *}$ \\ Bailing Wang ${ }^{3}$ \\ Zaohuo Cheng ${ }^{2}$ \\ Xingfu Zhao ${ }^{2}$ \\ Jianzhong Zhu ${ }^{2}$ \\ Degang Wang ${ }^{2}$ \\ Ying Wang ${ }^{2}$ \\ Aiguo Dong ${ }^{2}$ \\ Pengpeng $\mathrm{Li}^{2}$ \\ Chunhui Jin ${ }^{2}$}

'Mental Health Institute, The Second Xiangya Hospital, Central South

University, Changsha, Hunan Province,

${ }^{2}$ Wuxi Mental Health Center of

Nanjing Medical University, Wuxi,

Jiangsu Province, ${ }^{3}$ Qingdao Mental

Health Center, Qingdao, Shandong

Province, People's Republic of China

*These authors contributed equally to this work

\begin{abstract}
In previous studies, we reported that the sortilin-related receptor, L (DLR class) A repeats containing (SORL1) gene single nucleotide polymorphisms (SNPs) are associated with the risk of sporadic Alzheimer's disease (SAD) in the Han Chinese population. To further explore the relationships between SORL1 genetic variants and SAD, we conducted a two-step study. Sequencing analysis in 50 case samples identified 14 SNPs within the promoter and untranslated region of the SORL1 gene. Subsequent genotyping analysis in 106 patients with SAD and 179 healthy controls detected a significant association between the "G" allele of SNP rs 1133174 in the $3^{\prime}$ untranslated region of the SORL1 gene and SAD risk (odds ratio $=1.92,95 \%$ confidence interval $[95 \% \mathrm{CI}]=1.28-2.90$, adjusted $P=0.028$ ). In addition, "G" allele carriers of rs 1133174 $(\mathrm{GA}+\mathrm{GG})$ have a 2.15 -fold increased risk of SAD compared to noncarriers (AA) (adjusted $P=0.042$ ). However, no significant positive associations were observed in the other 13 SNPs within the SORL1 gene. These preliminary findings suggest that the SORL1 SNP rs1133174 may be a potential risk locus for SAD in the Han Chinese population.
\end{abstract}

Keywords: SORL1, Alzheimer's disease, polymorphism, Han Chinese, association

\section{Introduction}

Alzheimer's disease (AD) is an age-related progressive neurodegenerative disorder characterized by impairments in memory and other cognitive functions. ${ }^{1}$ Sporadic AD (SAD) affects the majority of patients with $\mathrm{AD}$, and it is a most common polygenic multifactorial disease suggested to be the result of interactions of genetic and environmental factors. ${ }^{2}$ Amyloid precursor protein (APP) and the presenilin genes 1 and 2 (PSEN1 and PSEN2) may be involved in early-onset familial AD, and currently, the $\varepsilon 4$ allele of the apolipoprotein E gene ( $A P O E \varepsilon 4)$ is the only well-established susceptibility gene for late-onset AD.

The sortilin-related receptor, L (DLR class) A repeats containing (SORL1, also called $L R 11$ or $\operatorname{sor} L A)$ gene, is a member of the low-density lipoprotein receptor family that reduces amyloid- $\beta(\mathrm{A} \beta)$ production by regulating the intracellular transport and processing of APP. ${ }^{3-5}$ Moreover, as an APOE receptor, SORL1 is a likely biomarker for AD because of its interaction with $A P O E$, A imaging study observed the effects of SORL1 variation on the brain microstructure during the neurodevelopmental phases of the human lifepan. ${ }^{6}$ Importantly, converging evidence from genetic and biological studies indicates that SORL1 could have an important role in AD susceptibility. ${ }^{7-9}$ Notably, actual functional risk loci within the SORL1 gene still remain unknown. Previous studies suggest that several rare and functional variants might play a crucial role in the development of AD. ${ }^{10,11}$ We reported that SORL1 genetic polymorphisms are significantly associated with 
susceptibility to SAD. ${ }^{12,13}$ To further explore the functional genetic variants within the SORL1 gene associated with SAD risk, we designed the present two-step study. We first sequenced the promoter, and the $5^{\prime}$ and $3^{\prime}$ untranslated regions (UTRs) in a total of 50 patients with SAD. All 14 single nucleotide polymorphisms (SNPs) were then genotyped and analyzed in the Han Chinese population.

\section{Materials and methods Study population}

A total of 50 patients with SAD, including 25 women and 25 men (75.6 \pm 9.8 years at recruitment), were assessed in the first step. In the genotyping step, the participants used in this case-control study included 106 unrelated patients ( 56 women and 50 men aged $76.4 \pm 10.5$ years at recruitment), and 179 unrelated healthy control subjects (96 women and 83 men aged $78.2 \pm 8.7$ years at recruitment). All participants were of Chinese Han descent and were recruited from the cities of Wuxi and Nanjing in Jiangsu province. A clinical diagnosis of probable AD was established according to the criteria of the National Institute of Neurological and Communicative Disorders and Stroke and the Alzheimer's Disease and Related Disorders Association. Healthy subjects were recruited through advertisement. Cognitive function was assessed by two skilled professional psychiatrists using the Mini-Mental State Examination. The demographic characteristics of the study participants are listed in Table 1.

The clinical study was in compliance with the World Medical Association Declaration of Helsinki - Ethical Principles for Medical Research Involving Human Subjects. The Ethics Committees of the Wuxi Health Mental Center approved this study. We excluded anyone who was not born in Jiangsu or whose family was not born in Jiangsu according to the self-reporting of their paternal grandparents and their place of birth. In addition, all patients and controls were not blood relations. Before enrollment in this study, all participants provided written consent forms.

Table I Demographic characteristics of the study subjects

\begin{tabular}{lll}
\hline & Control, $\mathbf{n}(\%)$ & SAD, $\mathbf{n}(\%)$ \\
\hline Total & 179 & 106 \\
Female & $96(53.6)$ & $56(52.8)$ \\
Male & $83(46.4)$ & $50(47.2)$ \\
Age, years & $78.2 \pm 8.7$ & $76.4 \pm 10.5$ \\
Age of onset & - & $71.1 \pm 9.7$ \\
Occupation & Retired & Retired \\
MMSE & $28.2 \pm 2.5$ & $16.1 \pm 6.4^{*}$ \\
\hline
\end{tabular}

Note: Values represent mean $\pm S D$ where noted, $* P<0.05$, SAD vs controls.

Abbreviations: MMSE, Mini-Mental State Examination; SAD, sporadic Alzheimer's disease; SD, standard deviation.

\section{DNA extraction}

Blood samples were collected from all participants using the $\mathrm{K}_{2}$ EDTA tubes, and a Blood Genotyping DNA Extraction Kit (Tiangen Biotech, Beijing, People's Republic of China) was used to extract genomic DNA from $150 \mu \mathrm{L}$ of peripheral blood. The DNA samples were then stored at $-80^{\circ} \mathrm{C}$ for genotype analysis.

\section{DNA sequencing}

We performed sequencing of the SORL1 gene (chromosome 11, NC_000011.10, GRCh38) including 2,500 bp in the promoter and $5^{\prime}$ UTR and 4,195 bp in the $3^{\prime}$-UTR using the genomic DNA of 50 patients with SAD. The purified polymerase chain reaction products were sequenced using the ABI3730XL genetic analyzer according to the manufacturer's instructions (Applied Biosystems, Foster City, CA, USA). The sequences were analyzed with LaserGene (DNASTAR, Inc., Madison, WI, USA) and Mutation Surveyor (Softgenetics, State College, PA, USA).

\section{SNP genotyping}

The genotypes of 14 SNPs were analyzed by Shanghai Biowing Applied Biotechnology Co., Ltd (www.biowing.com.cn) using the ligase detection reaction-polymerase chain reaction (LDR-PCR) method. ${ }^{13-15}$ Genomic DNA extracted from the clinical samples was first subjected to multiplex PCR to obtain a PCR product that included SNPs. This PCR product and the LDR probes were then subjected to a multiplex LDR reaction with a DNA sequencer to detect the products.

Table 2 Identified SNPs by sequencing the SORLI gene in 50 patients with SAD

\begin{tabular}{|c|c|c|c|c|}
\hline SNP no & $\begin{array}{l}\text { SNP in } \\
\text { dbSNP }\end{array}$ & Allele $e^{\ddagger}$ & $\begin{array}{l}\text { Position in } \\
\text { NC_000011.10 }\end{array}$ & $\begin{array}{l}\text { SNP } \\
\text { property }\end{array}$ \\
\hline $\mathrm{I}$ & rs57785427 & $\mathrm{A} / \mathrm{C}$ & 121449848 & 5'-UTR \\
\hline 2 & rs4935774 & $\mathrm{C} / \mathrm{T}$ & 121451045 & $5^{\prime}-$ UTR \\
\hline 3 & rs|4004I029 & $\mathrm{T} / \mathrm{G}$ & $12|45| \mid 28$ & $5^{\prime}$-UTR \\
\hline 4 & rs 189323512 & $\mathrm{C} / \mathrm{T}$ & 121629830 & $3^{\prime}$-UTR \\
\hline 5 & rs 10892762 & $\mathrm{G} / \mathrm{A}$ & 121630529 & $3^{\prime}$-UTR \\
\hline 6 & rsII70979|3 & $G / A$ & 121630583 & $3^{\prime}$-UTR \\
\hline 7 & rs 10790449 & $\mathrm{~T} / \mathrm{C}$ & 121630697 & $3^{\prime}$-UTR \\
\hline 8 & rsII33I74 & $\mathrm{G} / \mathrm{A}$ & 121631046 & $3^{\prime}$-UTR \\
\hline 9 & rsII3I497 & $\mathrm{C} / \mathrm{G}$ & 121632036 & $3^{\prime}$-UTR \\
\hline 10 & rs3087925 & $A / G$ & 121632059 & $3^{\prime}$-UTR \\
\hline II & rsII31499 & $\mathrm{A} / \mathrm{G}$ & 121632158 & $3^{\prime}$-UTR \\
\hline 12 & rs|47839956 & $\mathrm{G} / \mathrm{C}$ & 121632779 & $3^{\prime}$-UTR \\
\hline 13 & rs 183106460 & $\mathrm{G} / \mathrm{A}$ & 121633119 & $3^{\prime}$-UTR \\
\hline 14 & rs7793।594 & C/A & 121633472 & $3^{\prime}$-UTR \\
\hline
\end{tabular}

Note: $¥$ Minor allele/major allele.

Abbreviations: dbSNP, single nucleotide polymorphism database; SAD, sporadic Alzheimer's disease; SNPs, single nucleotide polymorphisms; UTR, untranslated region. 


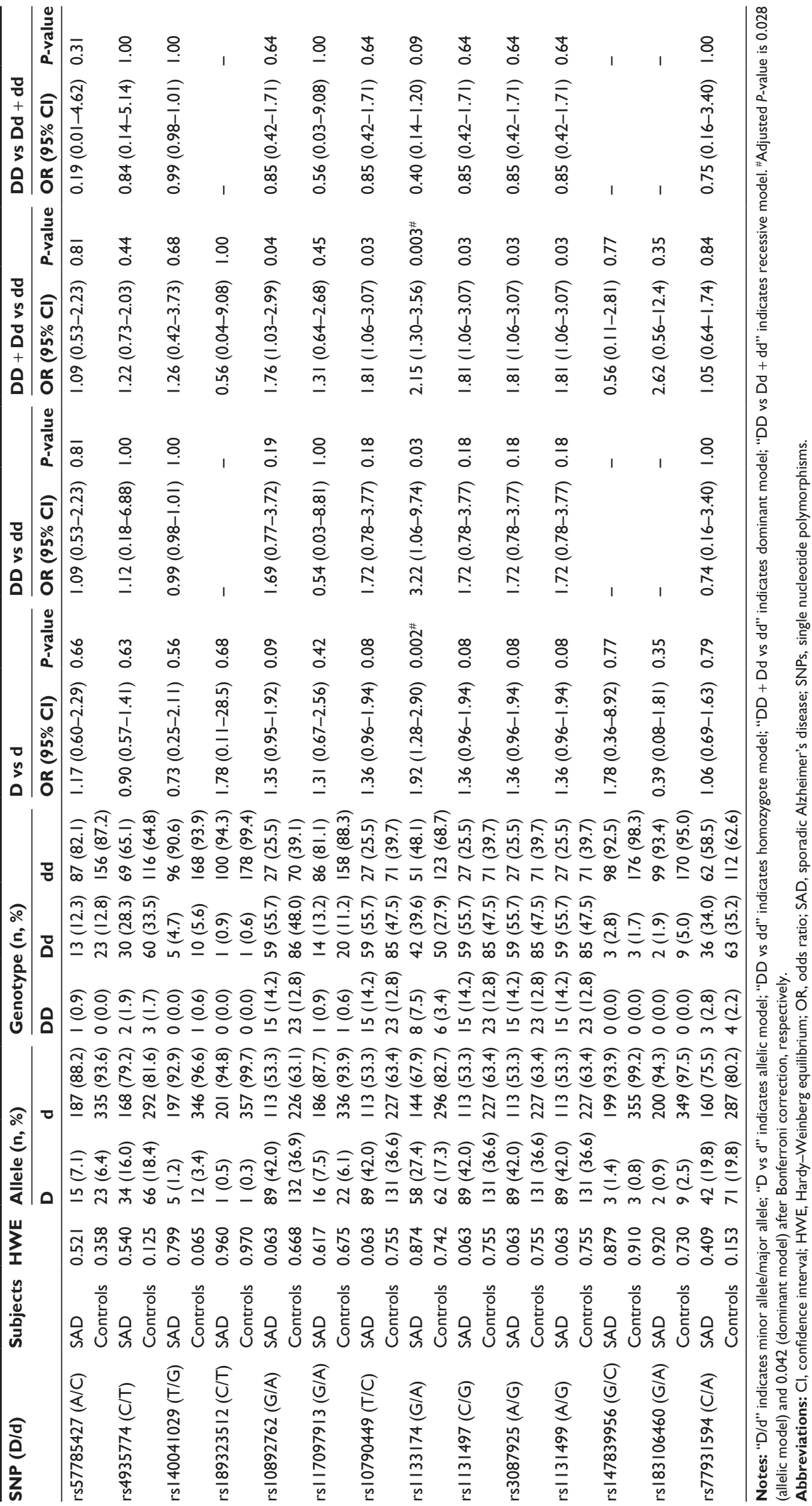


Table 4 Risk estimate using a logistic regression model for SORLI SNP rs I I 33 I 74

\begin{tabular}{llll}
\hline Group & Model & OR (95\% Cl) & $P$-value \\
\hline SAD & G vs A & $1.906(1.263-2.876)$ & 0.002 \\
& (GG + GA) vs AA & $2.129(1.287-3.52 I)$ & 0.003 \\
\hline
\end{tabular}

Note: a ${ }^{\mathrm{O} R}$ value is adjusted by sex and age.

Abbreviations: $\mathrm{Cl}$, confidence interval; OR, odds ratio; SAD, sporadic Alzheimer's disease; SNP, single nucleotide polymorphism.

The genotype call rate is $100 \%$ and $95 \%$ for controls and patients, respectively.

\section{Statistical analysis}

Our statistical analyses were performed using the PLINK (http://pngu.mgh.harvard.edu/ purcell/plink) and SPSS 17.0 software (IBM Corporation, Armonk, NY, USA) and included association studies, Hardy-Weinberg equilibrium (HWE) tests, and the calculation of genotype and allele frequencies in SAD and healthy control subjects. Haplotype analysis was conducted using the SHEsis software (http://analysis.bio-x. cn/myAnalysis.php/). ${ }^{16}$ Linkage disequilibrium was analyzed using the Haploview software (http://www.broadinstitute.org/ haploview/). Power calculations for our sample size were calculated using the PS software (http://biostat.mc.vanderbilt.edu/ wiki/Main/PowerSampleSize), ${ }^{17,18}$ and functional prediction was performed using the rSNPBase (rsnp.psych.ac.cn/). ${ }^{19}$

\section{Results}

A total of 14 SNPs were detected in the sequencing analysis, which were previously reported in the SNP database established by the National Center for Biotechnology Information (Table 2). Our HWE tests indicated that the allelic frequency distribution of the 14 SORL1 polymorphisms in the 106 patients with SAD and 179 healthy controls do not deviate significantly from HWE (Table 3 ). The power of the present association study is approximately $65 \%$ for higher frequency variants using the PS software (assumption condition: $\left.\alpha=0.05, P_{0}=0.2, \mathrm{n}=106, m=1.7, \Psi=2.0\right)$.

Our results suggest that there is a significant association of the " $G$ " allele of SNP rs1133174 in the 3'-UTR of the SORL1 gene and SAD risk (odds ratio $=1.92,95 \%$ confidence interval $=1.28-2.90$, adjusted $P=0.028$ ). Moreover, the "G" allele carriers of rs1133174 (GA + GG) have a 2.15-fold increased risk of SAD compared to noncarriers (AA; adjusted $P=0.042$ ) via a dominant model (Table 3 ). In addition, a logistic regression analysis revealed consistent results (Table 4). However, no significant positive associations were observed in the other 13 SNPs within the SORL1 gene. In addition, we constructed haplotype blocks using our own genotyping data for patients with SAD and controls (Table 3). Two complete linkage disequilibrium regions as well as the $D^{\prime}$ and $r^{2}$ values are provided (Figure 1). However, we failed to detect any positive haplotype signals in the present study (Table 5).

\section{Discussion}

Previous findings show that genetics variants within the SORL1 gene are significantly associated with AD susceptibility in the Han Chinese population. ${ }^{13,20-24}$ To identify new genetic variants within the SORL1 gene associated with SAD susceptibility in the Han Chinese population, we performed this two-step study. To our knowledge, this is the first study to associate the "G" allele within the SORL1 SNP

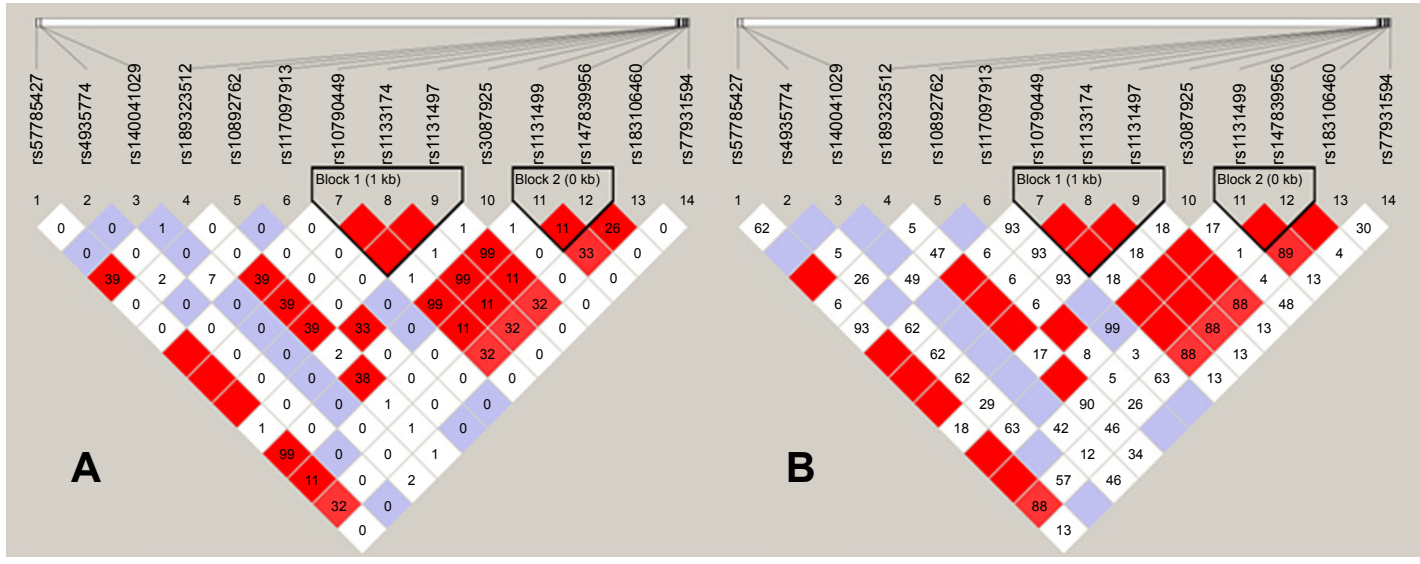

Figure I Linkage disequilibrium (LD) analyses for the SORLI gene generated from genotype data in I06 patients with SAD and I79 healthy controls.

Note: $r^{2}(\mathbf{A})$ and $D^{\prime}(\mathbf{B})$ LD values are shown separately.

Abbreviation: SAD, sporadic Alzheimer's disease. 
Table 5 Estimated haplotype frequencies in patients with SAD and control subjects

\begin{tabular}{|c|c|c|c|c|c|c|c|}
\hline Gene & Block & Haplotype & Case (freq) & Control (freq) & $\chi^{2}$ & $P$-value & OR (95\% CI) \\
\hline \multirow[t]{5}{*}{ SORLI } & rs|0790449-rs ||33|74-rs ||3|497 & $\mathrm{ACA}$ & $89(0.44)$ & $|3|(0.37)$ & 3.019 & 0.082 & $1.37(0.96-1.94)$ \\
\hline & & GCG & II $3(0.56)$ & $227(0.63)$ & 3.019 & 0.082 & $0.73(0.52-1.04)$ \\
\hline & rs||3|499-rs|47839956 & AA & $113(0.56)$ & $226(0.63)$ & 2.793 & 0.095 & $0.74(0.52-1.05)$ \\
\hline & & GA & $73(0.36)$ & $110(0.31)$ & 1.721 & 0.189 & $1.28(0.89-1.84)$ \\
\hline & & GG & $16(0.08)$ & $22(0.06)$ & 0.642 & 0.423 & I.31 (0.67-2.56) \\
\hline
\end{tabular}

Note: Haplotypes were omitted from analysis if the estimated haplotype probabilities were less than $3 \%$.

Abbreviations: $\mathrm{Cl}$, confidence interval; freq, frequency; OR, odds ratio; SAD, sporadic Alzheimer's disease.

rs1133174 with an increased risk of SAD in the Han Chinese population. The SNP rs1133174 is located in the 3'-UTR of the SORL1 gene. Usually, as a regulatory SNP (rSNP), rs1133174, would not influence the amino acid sequences or function of the protein directly. The results of the functional prediction from rSNPBase database suggest that the SORL1 SNP rs1133174 may be involved in transcriptional regulation and may affect the expression quantitative trait loci of multiple genes (data not shown). Importantly, a previous study indicated that the $S N P$ rs1133174 was significantly associated with hippocampal atrophy in older persons. ${ }^{25}$ The converging evidence suggests that the further functional investigation is warranted.

One purpose of the present study is to identify new uncommon variants within the SORL1 gene associated with the risk of AD. Several SNPs with lower frequencies (such as rs189323512, rs147839956, and rs183106460) were found during the sequencing stage, but no significant positive associations were observed. However, the relatively small sample size is a major limitation in the present study and may affect the interpretation of the results, particularly for rare variants (lower power). In addition, we reported that SORL1 SNP rs985421 might play a contrasting role in the Han Chinese compared to Japanese subjects, ${ }^{13}$ which suggests that populations from different geographic regions may exhibit different genetic markers for AD development. Although participants who were not born in Jiangsu or whose family was not born in Jiangsu were excluded, we could not completely eliminate the effect of different ethnic origins on the results, which is also a limitation of this study. As previously discussed, the SORL1 gene is a valuable susceptibility gene for $\mathrm{AD}$, whereas the actual functional variants associated with AD risk within the SORL1 gene remain unknown. Although the current results are preliminary, they provide new insights into the association of SORL1 with the risk of AD. Notably, AD is a multifactorial disease affected by both inherited and environmental factors, and $A P O E \varepsilon 4$ is the most prevalent genetic risk factor for SAD ${ }^{26}$ Therefore, follow-up studies with large-scale samples and stratified analyses of $A P O E \varepsilon 4$-status are necessary in the future.
In summary, this case-control study of the Han Chinese population suggests that an rSNP within the SORL1 gene, rs1133174, might affect SAD risk. However, the present findings and the association of rs1133174 with previously published risk loci must be further validated and investigated.

\section{Acknowledgments}

We thank all participants in this study. This work was supported by a grant from Wuxi Municipal Health Bureau Foundation (Q201208), Science and Technology Development Foundation of Nanjing Medical University (2013NJMU203), and Excellent Young Talents Project of Wuxi Municipal Health Bureau.

\section{Disclosure}

The authors report no conflicts of interest in this work.

\section{References}

1. Roses AD. The Alzheimer diseases. Curr Opin Neurobiol. 1996;6(5): 644-650.

2. Tanzi RE. The genetics of Alzheimer disease. Cold Spring Harb Perspect Med. 2012;2(10):a006296.

3. Andersen OM, Reiche J, Schmidt V, et al. Neuronal sorting protein-related receptor sorLA/LR11 regulates processing of the amyloid precursor protein. Proc Natl Acad Sci US A. 2005;102(38):13461-13466.

4. Andersen OM, Schmidt V, Spoelgen R, et al. Molecular dissection of the interaction between amyloid precursor protein and its neuronal trafficking receptor SorLA/LR11. Biochemistry. 2006;45(8):2618-2628.

5. Fjorback AW, Seaman M, Gustafsen C, et al. Retromer binds the FANSHY sorting motif in SorLA to regulate amyloid precursor protein sorting and processing. J Neurosci. 2012;32(4):1467-1480.

6. Felsky D, Szeszko P, Yu L, et al. The SORL1 gene and convergent neural risk for Alzheimer's disease across the human lifespan. Mol Psychiatry. 2014;19(10):1125-1132.

7. Gustafsen C, Glerup S, Pallesen LT, et al. Sortilin and SorLA display distinct roles in processing and trafficking of amyloid precursor protein. J Neurosci. 2013;33(1):64-71.

8. Rogaeva E, Meng Y, Lee JH, et al. The neuronal sortilin-related receptor SORL1 is genetically associated with Alzheimer disease. Nat Genet. 2007;39(2):168-177.

9. Tsolakidou A, Alexopoulos P, Guo LH, et al. Beta-Site amyloid precursor protein-cleaving enzyme 1 activity is related to cerebrospinal fluid concentrations of sortilin-related receptor with A-type repeats, soluble amyloid precursor protein, and tau. Alzheimers Dement. 2013;9(4):386-391.

10. Jonsson T, Stefansson H, Steinberg S, et al. Variant of TREM2 associated with the risk of Alzheimer's disease. N Engl J Med. 2013; 368(2):107-116. 
11. Steinberg S, Stefansson H, Jonsson T, et al. Loss-of-function variants in ABCA7 confer risk of Alzheimer's disease. Nat Genet. 2015;47(5):445-447.

12. Jin C, Liu X, Zhang F, et al. An updated meta-analysis of the association between SORL1 variants and the risk for sporadic Alzheimer's disease. J Alzheimers Dis. 2013;37(2):429-437.

13. Jin C, Zhang L, Xian Y, et al. The SORL1 polymorphism rs 985421 may confer the risk for amnestic mild cognitive impairment and Alzheimer's disease in the Han Chinese population. Neurosci Lett. 2014; 563:80-84.

14. Jin C, Zhang Y, Wang J, et al. Lack of association between MPC2 variants and schizophrenia in a replication study of Han Chinese. Neurosci Lett. 2013;552:120-123.

15. Shi Y, Li Z, Xu Q, et al. Common variants on 8p12 and 1q24.2 confer risk of schizophrenia. Nat Genet. 2011;43(12):1224-1227.

16. Li Z, Zhang Z, He Z, et al. A partition-ligation-combination-subdivision EM algorithm for haplotype inference with multiallelic markers: update of the SHEsis (http://analysis.bio-x.cn). Cell Res. 2009; 19(4):519-523.

17. Dupont WD, Plummer WD Jr. Power and sample size calculations. A review and computer program. Control Clin Trials. 1990; 11(2):116-128.

18. Dupont WD, Plummer WD Jr. Power and sample size calculations for studies involving linear regression. Control Clin Trials. 1998;19(6):589-601.

19. Guo L, Du Y, Chang S, Zhang K, Wang J. rSNPBase: a database for curated regulatory SNPs. Nucleic Acids Res. 2014;42(Database issue): D1033-D1039.
20. Feng X, Hou D, Deng Y, et al. SORL1 gene polymorphism association with late-onset Alzheimer's disease. Neurosci Lett. 2015; 584:382-389.

21. Gao X, Liu M, Sun L, et al. SORL1 genetic variants modulate risk of amnestic mild cognitive impairment in northern Han Chinese. Int J Neurosci. 2014;124(4):296-301.

22. Ning M, Yang Y, Zhang Z, et al. Amyloid-beta-related genes SORL1 and ACE are genetically associated with risk for late-onset Alzheimer disease in the Chinese population. Alzheimer Dis Assoc Disord. 2010;24(4):390-396.

23. Tan EK, Lee J, Chen CP, et al. SORL1 haplotypes modulate risk of Alzheimer's disease in Chinese. Neurobiol Aging. 2009;30(7): 1048-1051.

24. Xue X, Zhang M, Lin Y, Xu E, Jia J. Association between the SORL1 rs2070045 polymorphism and late-onset Alzheimer's disease: interaction with the ApoE genotype in the Chinese Han population. Neurosci Lett. 2014;559:94-98.

25. Assareh AA, Piguet O, Lye TC, et al. Association of SORL1 gene variants with hippocampal and cerebral atrophy and Alzheimer's disease. Curr Alzheimer Res. 2014;11(6):558-563.

26. Michaelson DM. APOE epsilon4: the most prevalent yet understudied risk factor for Alzheimer's disease. Alzheimers Dement. 2014;10(6):861-868.
Neuropsychiatric Disease and Treatment

\section{Publish your work in this journal}

Neuropsychiatric Disease and Treatment is an international, peerreviewed journal of clinical therapeutics and pharmacology focusing on concise rapid reporting of clinical or pre-clinical studies on a range of neuropsychiatric and neurological disorders. This journal is indexed on PubMed Central, the 'PsycINFO' database and CAS,

\section{Dovepress}

and is the official journal of The International Neuropsychiatric Association (INA). The manuscript management system is completely online and includes a very quick and fair peer-review system, which is all easy to use. Visit http://www.dovepress.com/testimonials.php to read real quotes from published authors. 